\title{
Erratum to: inferring the global structure of chromosomes from structural variations
}

\author{
Tomohiro Yasuda ${ }^{1,2}$ and Satoru Miyano ${ }^{1 *}$
}

\section{Corrections}

After publication of [1] we became aware that author revisions had not been incorporated into the final published version. The following corrections should be made. A PDF version into which all corrections are incorporated is attached as Additional file 1.

\section{Formatting}

Incorrectly formatted descriptions in [1] should be corrected as follows.

1. In the original publication, the images of Figures 1, 3, 5-9 are shuffled. In addition, images of Figures 1, 3, 4, 6-9 contain incorrectly encoded symbols. They should be replaced with Figures (1, 2, 3, 4, 5, 6, 7, 8, 9) presented in this article.

2. All four occurrences of "Yasuda and Miyano Page $n$ of 11 " in the main text should be removed.

3. All three occurrences of " $O\left(|E|_{2} \log |V| \log |E|\right)$ " should read " $O\left(|E|^{2} \log |V| \log |E|\right)$ "

4. In the Results, both of two "CC $(\tilde{G}, E+)$ " should read "CC $\left(\tilde{G}, E_{+}\right)$"

5. In the Methods, a Q.E.D. symbol “ $\square$ (white box)" should be inserted at the end of the following lines:

- The line just before Lemma 2

- The line just before Lemma 3

- The second line from below before "Proof of Theorem 2", ending with "a Hamiltonian cycle on H."

- The line with the sentence "Therefore, $C$ satisfies (6)." in subsection Circular chromosome graph

6. In the Background, "BCRABL" should read "BCR-ABL"
7. In the Methods, in the proof of Lemma 1, the expression " $c=p_{1} e_{1} p_{2} e_{2} \ldots e_{t} c p_{t} c_{+1}$ " should read "c $=p_{1} e_{1} p_{2} e_{2} \ldots e_{t_{c}} p_{t_{c}+1}$ "

8. In the same proof, just above Lemma 2, the expression "2 $|V|\left(n_{N}+n_{T}\right)+(4|V|+1) \mathrm{P}_{e} * * *_{E} S n(e) \leq U(4|V|+1)$ $(|E|+1)$ " should read " $2|V|\left(n_{N}+n_{T}\right)+(4|V|+1)$ $\sum_{e \in E_{S}} n(e) \leq U(4|V|+1)(|E|+1) "$

9. In the Methods, in the proof of Lemma 3, the following expressions

$$
\begin{aligned}
& e_{i, 1}=<-v_{i, 1}^{-},+v_{i, 2}^{\ddagger}, 1,1>\left(2 \leq i \leq\left|V^{\prime}\right|\right), \\
& e_{i, 2}=<-v_{i, 2}^{-},+v_{i, 3}^{\ddagger}, 0,1>\left(2 \leq i \leq\left|V^{\prime}\right|\right) .
\end{aligned}
$$

should read

$$
\begin{aligned}
& e_{i, 1}=<-v_{i, 1}^{-},+v_{i, 2}^{+}, 1,1>\left(2 \leq i \leq\left|V^{\prime}\right|\right), \\
& e_{i, 2}=<-v_{i, 2}^{-},+v_{i, 3}^{+}, 0,1>\left(2 \leq i \leq\left|V^{\prime}\right|\right) .
\end{aligned}
$$

10. In the Methods, in the proof of Lemma 4, in the paragraph that begins with "All of these steps", the expression " $m(C, e)=f(e)+f(\bar{e})\left(\in E_{S}\right)$." should read " $m(C, e)=f(e)+f(\bar{e})\left(e \in E_{S}\right)$." (Insert a white space before " $\left(e \in E_{S}\right)$ ".)

11. In the same proof, just before "Therefore, $C$ satisfies (6).", the expression " $w(e, m(C, e))=0\left(e \in E_{L} \cup E_{R}\right)$." should read " $w(e, m(C, e))=0 \quad\left(e \in E_{L} \cup E_{R}\right) . "$ (Insert a white space before " $\left(e \in E_{L} \cup E_{R}\right)$ ".)

\section{Inaccurate descriptions}

The following items correct inaccurate descriptions in the original manuscript. We regret any inconvenience that they might have caused.

1. In the Results, in subsection Formulation of the problem, the phrase "its computational complexity was not analyzed" should read "its computational complexity was not intensively analyzed"

\footnotetext{
*Correspondence: miyano@hgc.jp

${ }^{1}$ The Human Genome Center, Institute of Medical Science, University of Tokyo, Shiroganedai, Minato-ku, Tokyo, Japan

Full list of author information is available at the end of the article
} 

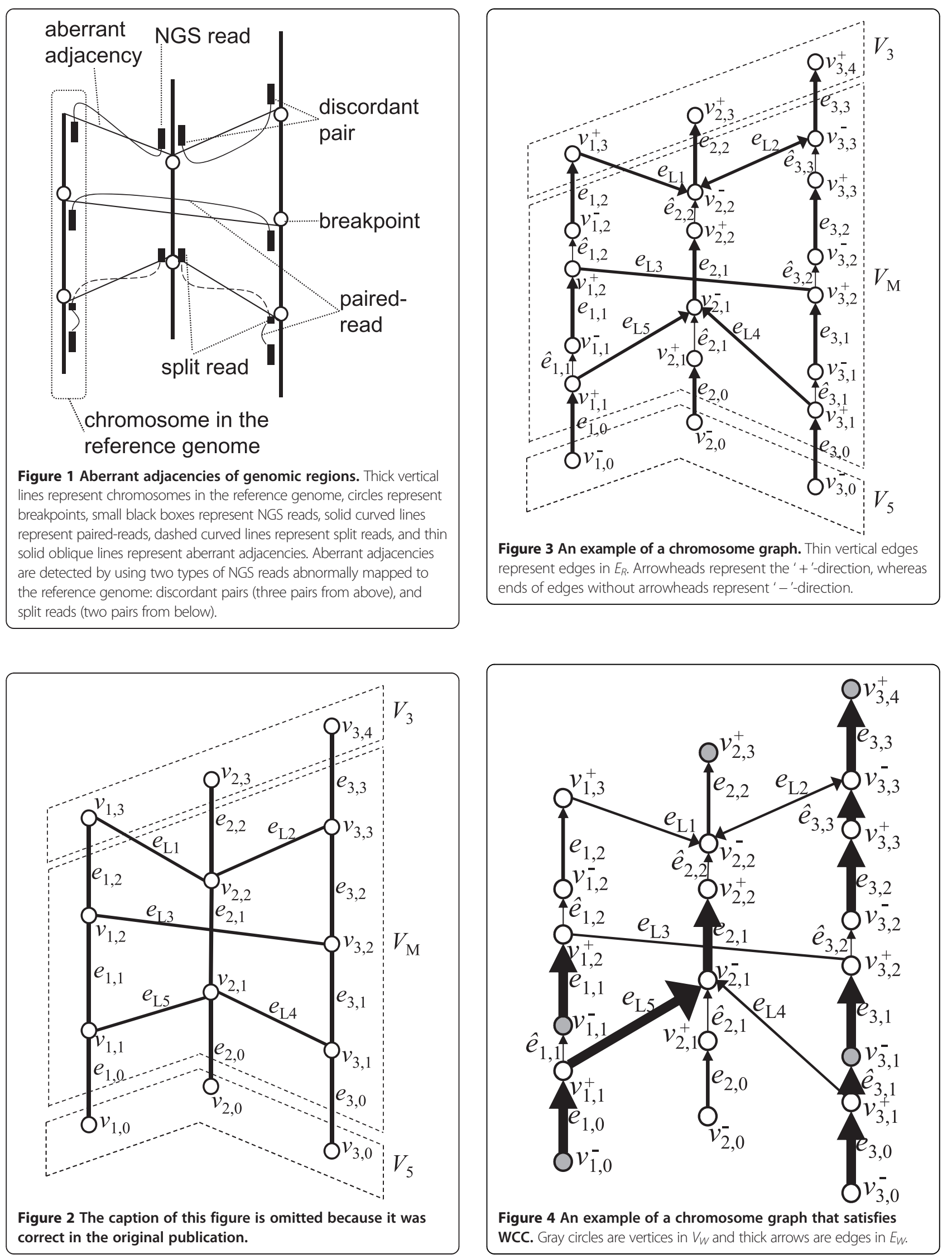

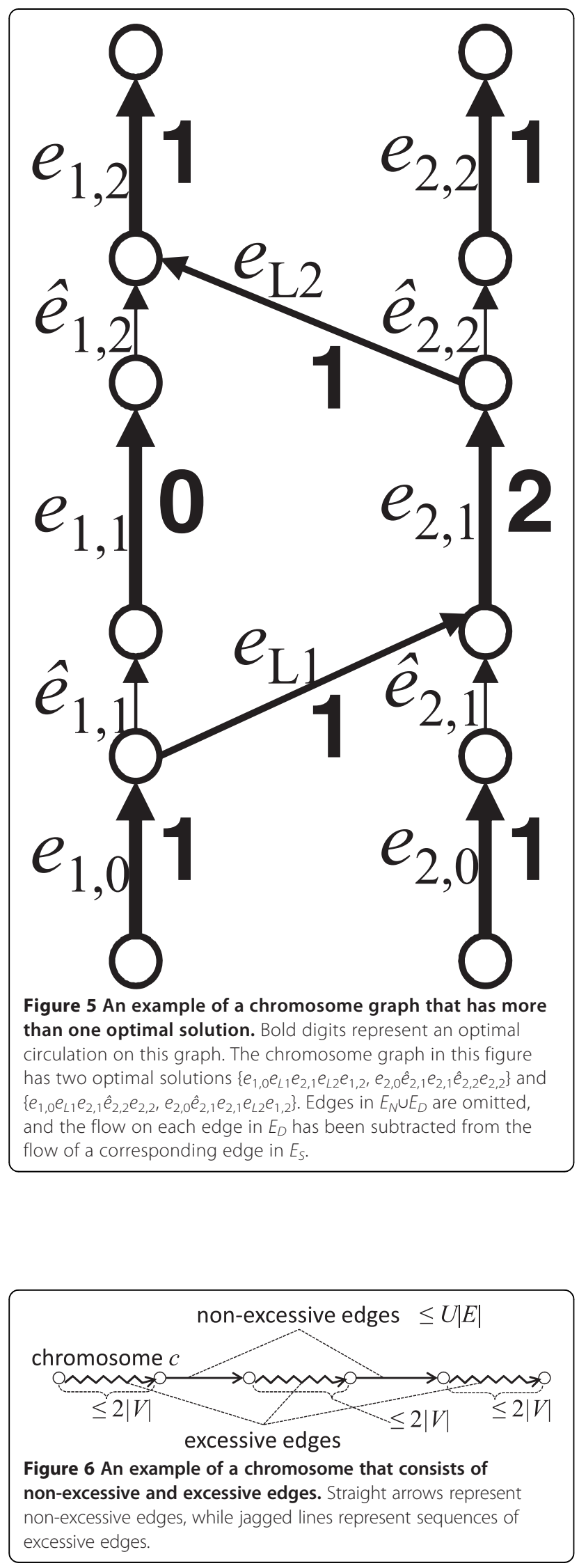

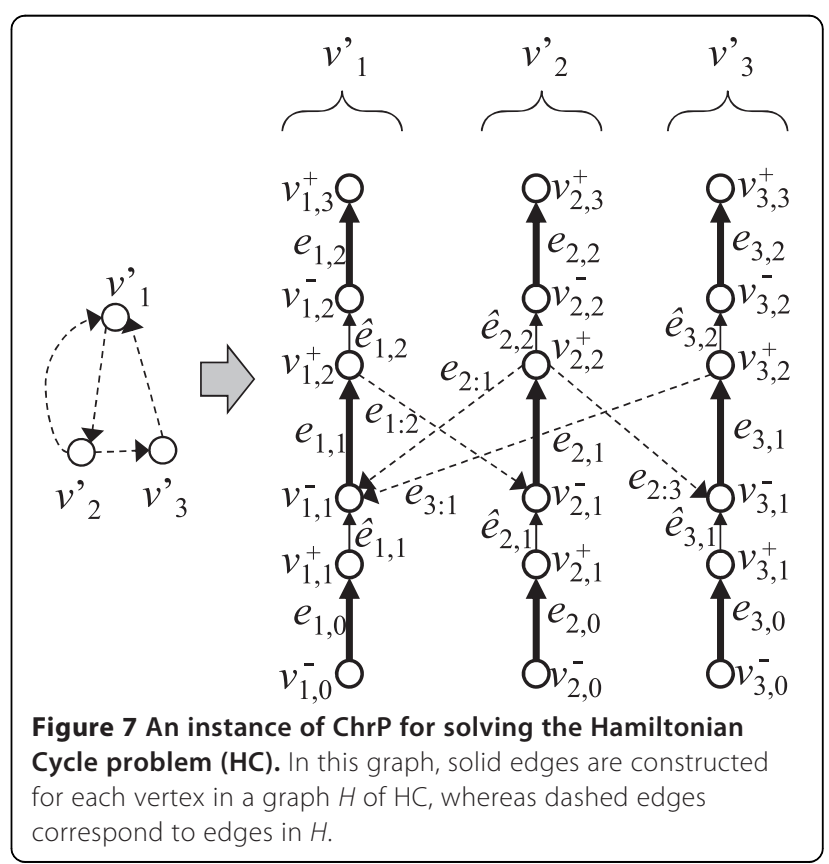

2. In the Results, in the first paragraph of subsection Polynomial-time solvable variation, both of two " $E_{L} \cup E_{R}$ " should read " $E$ "

3. In the Results, in Definition 2, the phrase "if all $g \in \mathrm{CC}\left(\mathrm{G}, E_{W}\right)$ are good" should read "if all $g \in \mathrm{CC}$ (G, $\left.E_{W}\right)$ are good and $n(e)=0$ for $e \in E-E_{W}$ "

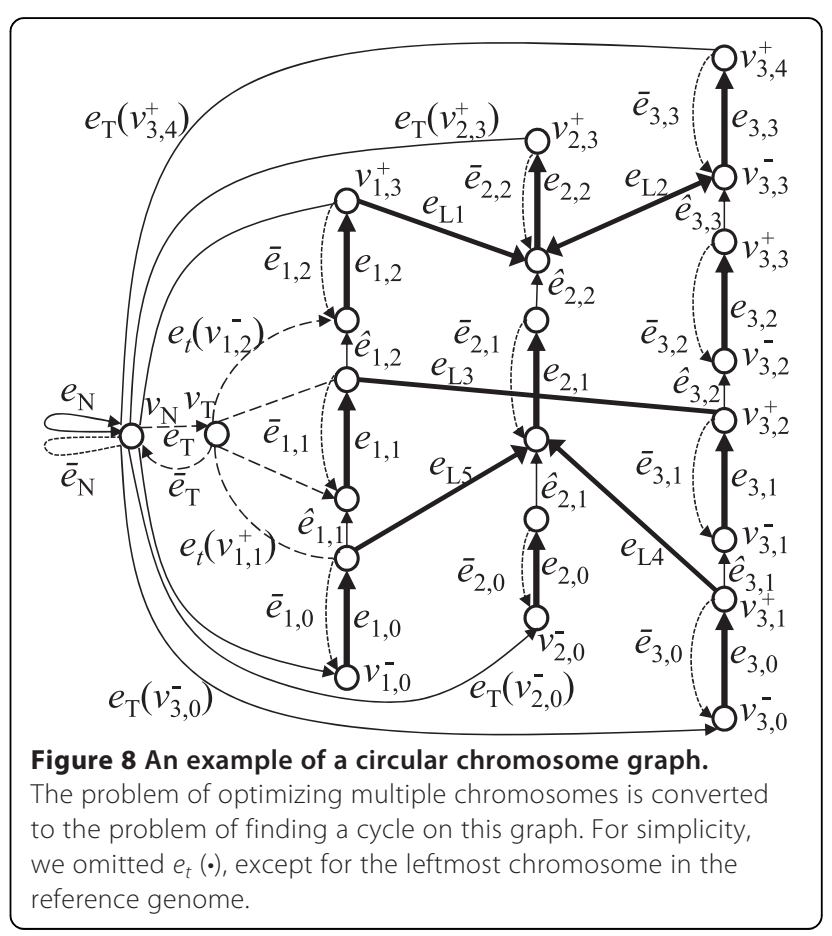




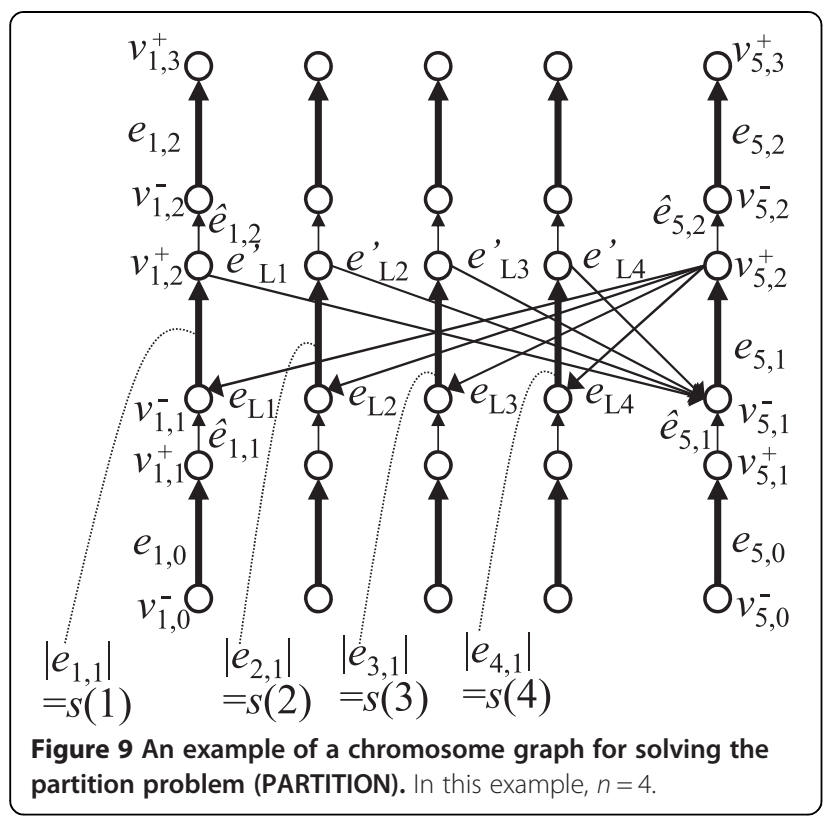

4. In the Results, in the paragraph just after Definition 2, the expression " $E_{W}=\left\{e \in E_{S} \mid n(e) \geq 1\right\} \cup\left\{e \in E_{L} \cup E_{R} \mid e\right.$ is required $\}$ " should read " $E_{W}=\{e \in E \mid e$ is required $\}$ "

5. In the Results, the last sentence that begins with "Finally, if some" just before Definition 3 should read as follows:

"In addition, if some $g \in C C\left(G, E_{W}\right)$ that are not good still remain, edges $e$ in $g$ are forcibly removed from $E_{W}$ by changing $e$ not required and setting $n(e)$ to 0 . Finally, if $n(e)>0$ for some $e \in E-E_{W}, e$ is changed to be required and added to $E_{W}$ by confirming its existence, or $n(e)$ is forcibly set to $0 . "$

6. In the Results, Definition 3 should read "Let $G=(V, E)$ be a chromosome graph that satisfies WCC with respect to given $V_{W} \subset \mathrm{V}$ and $E_{W} \subset E$. Then, find a set $C$ of chromosomes on $G$ that minimizes $W(C)$ when (3) is satisfied, each $v \in V_{W}$ is at an end of some $c \in C$, and each $e \in E_{W}$ appears in $C$."

7. In the Methods, in the paragraph just above Lemma 4 , the sentences "For $e \in E_{S} \cup\left\{e_{N}, e_{T}\right\}$, we set $l(e)=$ $n(e), l(\bar{e})=0$, and $u(\bar{e})=n(e)$. For $e \in E_{L} \cup E_{R}$, we set $l(e)=1$." should read "For $e \in E_{\mathrm{S}} \cup\left\{e_{N}, e_{T}\right\}$, we set $l(e)=n(e), l(\bar{e})=0$, and $u(\bar{e})=n(e)$ if $e$ is not required, whereas $l(e)=\max \{n(e), 1\}, l(\bar{e})=0$, and $u(\bar{e})=$ $\max \{n(e)-1,0\}$ if $e$ is required. We assume that $e_{N}$ is required because $n_{N} \geq 1$. We also assume that $e_{T}$ is required if $\left|V_{W}\right| \geq 1$. For $e \in E_{L} \cup E_{R}$, we set $l(e)=1$ if $e$ is required, or $l(e)=0$ otherwise."

8. In the Methods, in the paragraph just after Lemma 4, the description "or $n(e) \geq 1$ " should be removed.
9. In the Methods, in subsection Proof of Theorem 3, the phrase "by making all edges in $E_{L} \cup E_{R}$ required" should read "by making all edges required"

\section{Additional file}

Additional file 1: Corrected version. A PDF version of [1] into which all corrections in this article are incorporated.

\section{Author details}

${ }^{1}$ The Human Genome Center, Institute of Medical Science, University of Tokyo, Shiroganedai, Minato-ku, Tokyo, Japan. ${ }^{2}$ Department of Computer Science, University of Tokyo, Hongo, Bunkyo-ku, Tokyo, Japan.

Received: 11 February 2015 Accepted: 11 February 2015 Published: 9 April 2015

\section{Reference}

1. Yasuda T, Miyano S. Inferring the global structure of chromosomes from structural variations. BMC Genomics. 2015;16 Suppl 2:13.

\section{Submit your next manuscript to BioMed Central and take full advantage of:}

- Convenient online submission

- Thorough peer review

- No space constraints or color figure charges

- Immediate publication on acceptance

- Inclusion in PubMed, CAS, Scopus and Google Scholar

- Research which is freely available for redistribution 